\title{
Development and Psychometric Evaluation of an Instrument to Assess Knowledge, Attitude and Practice of Family Caregivers at Preventing Pressure Injuries (KAP-PI) in Indonesian Community-Dwelling Older Adults
}

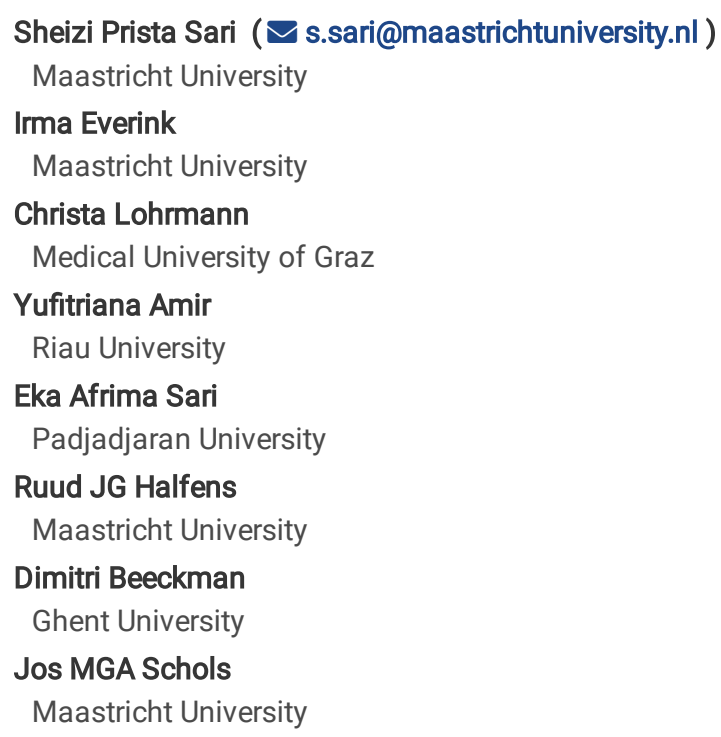

Keywords: Knowledge, Attitude, Practice, Pressure injury/ Pressure ulcer, Prevention, Family caregivers, Psychometric evaluation, Community nurses Posted Date: July 7th, 2021

DOI: https://doi.org/10.21203/rs.3.rs-668940/v1

License: (c) (i) This work is licensed under a Creative Commons Attribution 4.0 International License. Read Full License 


\section{Abstract}

Background: The prevalence of pressure injuries among community-dwelling older adults in countries worldwide is still a serious problem. In Indonesia, older adults mostly rely on family members for (medical) care. Therefore, involving family members in the prevention and treatment of Pls could potentially decrease its prevalence rates. However, family members are usually not trained for such tasks. Hence, it is essential to first get more insight into the current state of affairs on family members' knowledge, attitude and actual practice of preventing Pls. Due to the lack of an existing instrument to measure knowledge, attitude and practice of family caregivers in preventing Pls, this study focuses on the development and evaluation of psychometric properties of such an instrument.

Methods: Three phases of instrument development and evaluation were used, including item generation, instrument construction and psychometric testing of the instrument. A total of 372 family caregivers of community-dwelling older adults who randomly selected participated in this study. Statistical analysis of exploratory analysis, confirmatory factor analysis and Cronbach's alpha were used for the evaluation.

Results: The final version of the KAP-PI-instruments consists of a 12-item knowledge domain, a 9-item attitude domain, and a 12-item practice domain with Cronbach's Alpha values of $0.83,0.93$ and 0.89 , respectively. The instrument appeared to be both reliable and valid.

Conclusion: The KAP-PI instrument can be used in family nursing or community nursing practice, education, and research to assess knowledge, attitude and practice of pressure injury prevention of family caregivers.

\section{Introduction}

Research has shown that the prevalence of pressure injuries (PIs) among community-dwelling older adults in countries around the world is still a serious problem (1-3). The European Pressure Ulcer Advisory Panel, National Pressure Injury Advisory Panel and Pan Pacific Pressure Injury Alliance (2019) defined that "A pressure injury is a localized damage to the skin and or underlying tissue, which usually occurs over a bony prominence due to pressure or pressure combined with shear" (1). A study in the United States found that patients who had a PI on admission to the hospital were dominated (70.6\%) by older adults (mean age 72.7 years) living at home (2). Another study in the United States noted that PI were associated with greater number of older adult living at home re-admissions to the hospital (3). Similarly, a study in United Kingdom reported numbers of community-dwelling older adults with pressure injuries per 1000 were 1.64 for those aged $65-74$ and 5.75 for aged $\geq 74$ (4).

Indonesia is a country that is also facing challenges related to Pls, due to its ageing population (5). Indonesia has a population of 273.5 million people $(6,7)$, of which almost $10 \%$ are older adults $(60+)$ and the majority of these older adults live at home with their families $(8)$. Nursing homes or other long-term care institutions do exist, but are in minimal number $(8,9)$. Also, leaving parents in nursing homes is still taboo for Indonesian people (10). Older adults who live at home can also receive community care, but it appears that this formal care option is also not being used very often $(5,8)$. Therefore, family members are often the ones taking care of older adults with care dependency. They not only take care of activities of daily living their relatives need, such as washing and dressing, but also of more complex (medically-oriented) tasks, such as preventing and treating PIs $(5,11,12)$.

Involving family members in the prevention and treatment of Pls could potentially decrease its prevalence rates (13-16), especially among older adults living at home with a high risk of developing PIs. These are mainly older adults with limited mobility $(5,17,18)$, a stroke history $(5,19)$, and nutritional problems (20). However, little is known about what family members actually know about PI.

Preventing and treating Pls is a complex task, and family members are usually not trained for such tasks $(5,17,21)$. Besides the fact that not much is known about the knowledge family members have about PIs, less is also known about the attitude of family caregivers towards PI prevention and treatment. Knowledge and attitude appear to be positively associated with the actual practice of preventing illnesses (22-25).

To decrease prevalence of PIs in Indonesian community-dwelling older adults, it seems that a strategy focused on their family caregivers could be beneficial. However, to develop a targeted strategy, it is essential to first get more insight into the current state of affairs on family members' knowledge, attitude and actual practice of preventing Pls. To assess this, a valid and reliable instrument is needed. After a literature search focused on finding a standardized or published instrument that measures family caregivers' knowledge, attitude, and practice on PI prevention, it appeared that hardly any information was available. Only one study was found that reported on the psychometric properties of an instrument measuring knowledge on PI prevention (26).

Due to the lack of an existing instrument to measure knowledge, attitude and practice of family caregivers in preventing PIs, the objective of this study was to develop such an instrument and to describe its psychometric properties. In this study, the steps that were taken to develop and test the instrument are described, as well as the content of the final version of instrument for use in a population of community-dwelling older adults in Indonesia.

\section{Objective}


This study describes the development and psychometric evaluation of an instrument to measure Knowledge, Attitude and Practice of family caregivers to prevent PIs among community-dwelling older adults in Indonesia (KAP-PI).

\section{Method}

Three phases of instrument development and evaluation described by Boateng et al. (2018) were used, including 1) item generation; 2) instrument construction, and 3) psychometric testing of the instrument (27). Figure 1 describes the entire process of developing and psychometric evaluation of KAPI-PI instrument.

\subsection{Phase I: Item generation}

\section{Item Generation}

First, we specified the three domains we wanted to measure: knowledge $(K)$, attitude $(A)$, and practice $(P)$ of family caregivers in preventing PIs among community-dwelling older adults in Indonesia. To develop items covering the three domains, authors SPS and EAS conducted a literature search. They independently identified relevant topics for measuring family caregivers' knowledge, attitudes, and practices for preventing PIs in community-dwelling older adults. The literature review was conducted using the following questions: 1) What is the necessary information about PI prevention that family caregivers should know; 2) What attitude toward PI prevention should family caregivers have; and 3) What should family caregivers actually do to prevent their older relative from getting a PI? After a detailed review of the international PI guideline developed by the National Pressure Ulcer Advisory Panel (NPUAP), The European Pressure Ulcer Advisory Panel (UPUAP) and Pan Pacific Pressure Injury Alliance (PPPIA) (28), the two authors compared and merged their findings. Considering that the guideline is written primarily for health professionals in clinical practice, a review of family nursing books (29-31) was also conducted to narrow the findings in the family care function, resulting in the themes per domain shown in Fig. 1 .

After determining the topics in the three domains, the authors proceeded to generating questions per topic. The design of the questions followed the questionnaire design guide explained by Bourke et al. (32). For the items in the knowledge domain, multiple-choice questions with only one correct answer were developed (33). For the items in the attitude domain, statements were developed with response options on a Likert scale ranging from 1 (strongly disagree) to 4 (strongly agree) (34). Statements were also developed in the practice domain. An example is "I help the older relative to move when he is bedridden". The statements in the practice domain also include a response option on a Likert scale including the answer options 'never' (the activity has never been done), 'sometimes' (the activity is done 1 to 3 days per week), 'often' (the activity is done 4 to 6 days per week), and 'always' (the activity is done every day).

\section{Content and Face Validity Assessment}

Two nurses and one physician from Indonesia who have experience in the field of pressure injuries and community care were asked to evaluate the content of the instrument. Experts received the draft instrument via email and were asked to rate 1) the clarity of wording per item and 2) the relevance of each item per domain (knowledge, attitude, and practice) to the population under consideration (family caregivers caring for community-dwelling older adults). They were asked to rate the items on a 4-point Likert scale as follows:

1. How do you assess the relevance of topics and items in the three domains for the population under consideration? Answer options included $1=$ not relevant, 2 = somewhat relevant, 3 = quite relevant, 4 = highly relevant.

2. How do you assess the clarity of wording of this item? Answer categories were: $1=$ drop item entirely, $2=$ make major revisions to the item, $3=$ make minor revision to the item, 4 = retain the item exactly as worded.

If experts felt that the wording was not clear or the item was not relevant, they were asked to suggest for improvements. In addition, the experts were allowed to add topics or points which, in their opinion, were still missing in the instrument. The item content validity index (I-CVI) was calculated to evaluate the individual items in the instrument. The I-CVI is computed as the number of experts giving a rating of either 3 (quite relevant) or 4 (highly relevant), divided by the number of experts (35). Only if the I-CVI was 1.00, meaning that all experts gave a rating 3 (quite relevant) or 4 (highly relevant) on an item, the item was left in the instrument. This meant that the Instrument content validity index (S-CVI), calculated as the proportion of items from the original instrument rated as 'quite relevant' or 'highly relevant' by the experts was also 1 (reflecting excellent content validity) (35).

Next, face validity was assessed in the target population by including 12 family caregivers of community-dwelling older adults. This process aimed to confirm that family caregivers understood the questions as the researchers intended. They were first asked to fill out the instrument. Then, in a short interview, family caregivers were asked whether they recognized every word used on the questionnaire and understood the meaning of the question or not. Correction of sentences and word choices were made based on their feedback.

The content and face validity process resulted in a preliminary KAP-PI instrument.

\subsection{Phase II: Instrument construction}

Page $3 / 24$ 
Phase II aimed to transform the preliminary KAP-PI instrument into a statistically acceptable construct (27). At this stage, data were collected using a self-administration paper-based instrument and used to determine: 1) which items should be deleted; and 2) the optimal number of factors that fit a set of items (27).

\section{Participants}

For phase II, family caregivers were required to complete the preliminary version of the KAP-PI. For this, family caregivers of community-dwelling older adults in all regions in Bandung, Indonesia, were randomly selected from a list of family data provided by the municipalities. To be included in the study, family caregivers (spouses, children, or other relatives of older adults) had to live with or care for people aged 60 years or older (considered older adults in Indonesia (36)). From each selected family, one family caregiver filled out one instrument, irrespective of the number of the older adult in him/ her household. The number of participants needed in total for the statistical analysis purposes was determined by 10 participants per survey item or 200-300 participants (27).

\section{Data collection}

Selected family caregivers were visited in their home by enumerators, who were independently recruited as research assistants. If they met the inclusion criteria and agreed to participate, data collection immediately took place in the participant's home. Participants received an informed consent form and the Preliminary KAP-PI paper-based instrument consisting of demographic data, questions and instructions. The enumerators first explained the study's objectives, the procedure, the anonymized use of data and the right to stop participation at any moment. Participants completed the self-administration of preliminary KAP-PI instrument in front of the enumerators. The completed instruments were collected by the enumerators and given to the authors.

\section{Data analysis}

\section{Knowledge domain}

The answers to the questions, which consisted of multiple-choice questions, were recorded in dichotomous correct-incorrect variables. Correct answers were assigned the value 1 , incorrect answers the value 0 . Instrument construction of the knowledge domain was based on an evaluation described by Haladyna (33), which includes the evaluation of 1 ) the item difficulty; 2 ) the discriminating index (D value); and 3 ) the quality of response alternatives. The decision to delete some items was based on these ratings as explained below:

The item difficulty is the percentage of participants who answer the item correctly (33). Items with a lower item difficulty are relatively easier to answer compared to items with a higher difficulty score. In this study, items answered correctly by less than $10 \%$ of the participants were considered too difficult and item answered correctly by more than $90 \%$ of the participants were considered too easy. Hence, items with a difficulty index lower than 0.10 or higher than 0.90 were removed

The discriminating index ( $D$ value) describes an item's ability to differentiate between participants who know and do not know the information being asked. A statistical method of Item-total correlation (point-biserial) was used to evaluate the discrimination index of each item. Further, Cronbach's Alpha was checked. Items with item-total correlation being much lower than those of the other items and not contributing to internal consistency (i.e. alpha if item deleted > alpha with item in the scale) were deleted (33).

Finally, the quality of the response alternatives (that is, the distractors/wrong answers) was assessed by the proportion or percentage of participants who chose these distractors (range $0-1$ ) (33). The distractors with a value of 0 were defined as 'not attractive', and those with a value of 1 as 'too attractive'.

A study of dimensionality from the valid items in the knowledge domain was performed using a Principal Factor Analysis (PFA) with oblique rotation method with Kaiser Normalization.

\section{Attitude and practice domain}

In the attitude and practice domains, the answers to the questions were scored according to each answer's value, which ranged from 1 to 4 . A Principal Factor Analysis (PFA) with oblique rotation method with Kaiser Normalization was run separately for the two domains. This was done to check the relationship between the items and to check how many factors were generated from the items $(37,38)$.

Before performing the factor analysis, all requirements for performing PFA were checked, including 1) value of Kaiser-Meyer-Olkin Measure from Sampling Adequacy (KMO MSA) should be 0.50; 2) Bartletts Test of sphericity (Sig.) should be 0.05 (39). The number of factors in the attitude and

Page $4 / 24$ 
practice domains were extracted using Eigenvalues $>1$ and the total variance explained by the factors (39). A factor loading cutoff value of 0.4 was used to indicate the acceptable construct validity of each item: items with a value of 0.4 and higher were retained in the instrument (37).

All analyses in phase II were done using IBM SPSS Statistics 26 (IBM Corp, Armonk, NY).

\subsection{Phase III: Psychometric testing of the instrument}

\section{Participants and procedure}

Phase III tested the final KAP-PI instrument to different family caregivers in the same population with phase II. The inclusion criteria and handling of the data collection procedure for phase III were the same as phase II. The number of participants needed was determined by 10 participants per survey item or 200-300 participants (27).

\section{Data analysis}

In the knowledge domain, data analysis aimed to confirm the dimensionality of items. All the valid items were expected to form a unidimensional construct. In the attitude and practice domains, data analysis aimed to confirm number of factors constructed from phase II. A confirmatory factor analysis (CFA) was performed using the statistical computing $\mathrm{R}$ package lavaan from the Comprehensive R Archive Network (CRAN) (40, 41). The $\mathrm{R}$ package lavaan generates "fit indices" including Tucker-Lewis Index/ TLI (the higher the value, the better the model), Root Mean Square Error of Approximation/ RMSEA (is expected to be small to indicate reasonable model fit), and Comparative Fit Index/ CFI (value above 0.90 is considered good) (42). When the indices are fits, the correct model has been specified (42).

Lastly, the instrument's reliability (internal consistency) in each domain and per item subset were analyzed using Cronbach's alpha inter-item correlation. A general guideline for the use of Cronbach's alpha to assess a newly developed instrument is that values should be $\geq 0.70$ ( 43 ).

\section{Results}

\section{Characteristics of Participants}

Table 1 shows the participant characteristics. A total of 372 family caregivers participated in the study (12 participants in Phase I to investigate face validity, 120 participants in Phase II for instrument development, and 240 participants in Phase III for psychometric testing). Of all participating family caregivers, $89.0 \%(n=331)$ were female, and $69.1 \%(n=257)$ were $>30$ years old. Most participants $(n=307 ; 82.5 \%)$ had a low educational background (below upper secondary school education), and $38.9 \%(n=144)$ were unemployed. More than half $(n=266 ; 71.5 \%)$ of the caregivers were children of older adults. 
Characteristics of Participants

\begin{tabular}{|c|c|c|c|c|}
\hline \multirow[t]{2}{*}{ Characteristics of Participants } & \multicolumn{4}{|l|}{ Participants (\%) } \\
\hline & Face validity $(n=12)$ & Phase II $(n=120)$ & Phase III $(n=240)$ & $\begin{array}{l}\text { Total } \\
(n=372)\end{array}$ \\
\hline \multicolumn{5}{|l|}{ Gender } \\
\hline Male & $3(20.0)$ & $15(12.5)$ & $23(9.6)$ & $41(11.0)$ \\
\hline Female & $9(80.0)$ & $105(87.5)$ & $217(90.4)$ & $331(89.0)$ \\
\hline \multicolumn{5}{|l|}{ Age category } \\
\hline$<20$ years & $0(0.0)$ & $7(5.8)$ & $14(5.8)$ & $21(5.6)$ \\
\hline $20-30$ years & $3(20.0)$ & $28(23.3)$ & $63(26.3)$ & $94(25.3)$ \\
\hline $31-40$ years & $6(60.0)$ & $33(27.5)$ & $54(22.5)$ & $93(25.0)$ \\
\hline$>40$ years & $3(20.0)$ & $52(43.3)$ & $109(45.4)$ & $164(44.1)$ \\
\hline \multicolumn{5}{|l|}{ Education } \\
\hline Primary education & $3(30.0)$ & $82(68.3)$ & $147(61.3)$ & $232(62.4)$ \\
\hline Lower secondary education & $2(10.0)$ & $15(12.5)$ & $58(24.2)$ & $75(20.2)$ \\
\hline Upper secondary education & $7(60.0)$ & $16(13.3)$ & $27(11.3)$ & $50(13.4)$ \\
\hline Diploma & $0(0.0)$ & $7(5.8)$ & $8(3.3)$ & $15(4.0)$ \\
\hline \multicolumn{5}{|l|}{ Occupation } \\
\hline Unemployed & $4(40.0)$ & $48(40.0)$ & $92(38.3)$ & $144(38.7)$ \\
\hline Student & $0(0.0)$ & $2(1.7)$ & $3(1.3)$ & $5(1.3)$ \\
\hline Employee & $2(20.0)$ & $38(31.7)$ & $84(35.0)$ & $124(33.3)$ \\
\hline Self-employed & $6(40.0)$ & $32(26.7)$ & $61(25.4)$ & $99(26.6)$ \\
\hline \multicolumn{5}{|l|}{ Relationship with older adult } \\
\hline Spouse & $0(0.0)$ & $17(14.2)$ & $28(11.7)$ & $45(12.1)$ \\
\hline Children & $6(50.0)$ & $63(52.5)$ & $170(70.8)$ & $239(64.2)$ \\
\hline Other relatives & $6(50.0)$ & $40(33.3)$ & $42(17.5)$ & $88(23.7)$ \\
\hline
\end{tabular}

\section{Knowledge domain}

Table 2 shows the topics and items generated in the knowledge domain and their statistical analysis results at each stage. In the process of item development, four topics and 18 items were generated (items no. 1-18). In the process of content validation, of these 18 items, five items (item no. $2,5,9,12$, and 18) were deleted due to a Content Validity Index (CVI) of < 1 each, while one item was added to the supporting interface (item no. 19). Thus, 14 items remained content valid and therefore, were tested for construct validity in phase II. 
Table 2

Knowledge domain

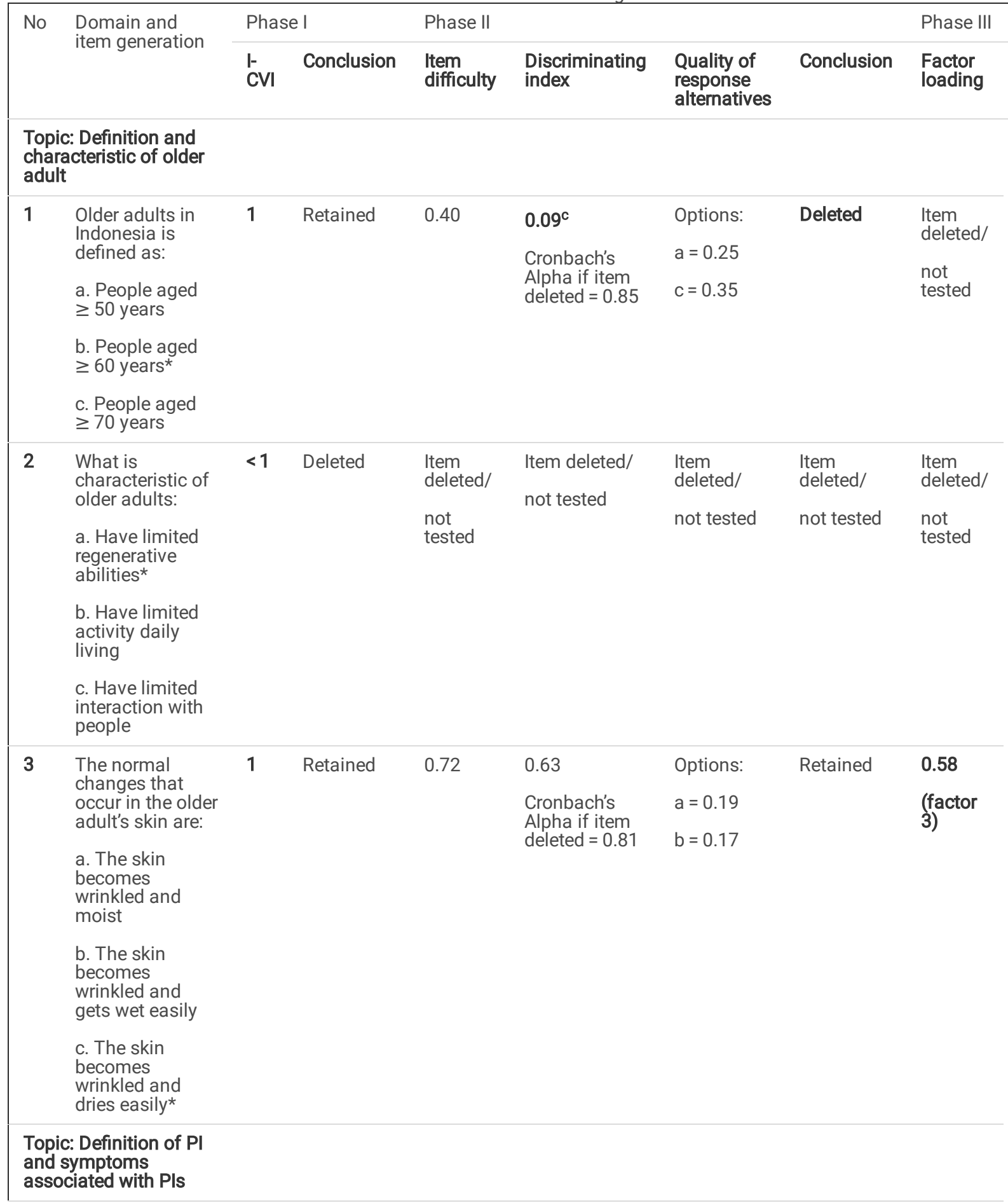

Note :

* correct answers

${ }^{a}$ Value of person correlation (r-value) smaller than r-table

b Value of item difficulty smaller than 0.10 or larger than 0.90

${ }^{c}$ Value of discriminant index (D value) smaller than 0.40 


\begin{tabular}{|c|c|c|c|c|c|c|c|c|}
\hline 4 & $\begin{array}{l}\text { A Pressure injury } \\
\text { is: } \\
\text { a. An injury that } \\
\text { occurs due to the } \\
\text { use of diapers } \\
\text { b. An injury on } \\
\text { the skin which } \\
\text { usually occurs } \\
\text { over a bony } \\
\text { prominence as a } \\
\text { result of } \\
\text { pressure* } \\
\text { c. An injury that } \\
\text { occurs due to } \\
\text { pressed by tight } \\
\text { clothes }\end{array}$ & 1 & Retained & 0.30 & $\begin{array}{l}\text { Cronbach's } \\
\text { Alpha if item } \\
\text { deleted }=0.82\end{array}$ & $\begin{array}{l}\text { Options: } \\
a=0.50 \\
c=0.20\end{array}$ & Retained & $\begin{array}{l}\text { (factor } \\
\text { 3) }\end{array}$ \\
\hline 5 & $\begin{array}{l}\text { A pressure injury } \\
\text { can also be } \\
\text { defined as: } \\
\text { a. An injury on } \\
\text { the skin over a } \\
\text { bony } \\
\text { prominence as a } \\
\text { result of shear } \\
\text { b. An injury on } \\
\text { the skin because } \\
\text { of heat } \\
\text { c. An injury on } \\
\text { the skin because } \\
\text { of diabetic }\end{array}$ & $<1$ & Deleted & $\begin{array}{l}\text { Item } \\
\text { deleted/ } \\
\text { not } \\
\text { tested }\end{array}$ & $\begin{array}{l}\text { Item deleted/ } \\
\text { not tested }\end{array}$ & $\begin{array}{l}\text { Item } \\
\text { deleted/ } \\
\text { not tested }\end{array}$ & $\begin{array}{l}\text { Item } \\
\text { deleted/ } \\
\text { not tested }\end{array}$ & $\begin{array}{l}\text { Item } \\
\text { deleted/ } \\
\text { not } \\
\text { tested }\end{array}$ \\
\hline 6 & $\begin{array}{l}\text { Symptom(s) of } \\
\text { pressure injuries } \\
\text { are: } \\
\text { a. The skin looks } \\
\text { reddish } \\
\text { b. There is } \\
\text { visible skin } \\
\text { damage/wounds } \\
\text { c. Options A and } \\
\text { B are correct* }\end{array}$ & 1 & Retained & 0.72 & $\begin{array}{l}0.68 \\
\text { Cronbach's } \\
\text { Alpha if item } \\
\text { deleted }=0.80\end{array}$ & $\begin{array}{l}\text { Options: } \\
a=0.18 \\
b=0.10\end{array}$ & Retained & $\begin{array}{l}0.42 \\
\text { (factor } \\
3 \text { ) }\end{array}$ \\
\hline \multicolumn{9}{|c|}{$\begin{array}{l}\text { Topic: Cause and } \\
\text { consequences of Pls }\end{array}$} \\
\hline 7 & $\begin{array}{l}\text { The cause of a } \\
\text { pressure injury } \\
\text { is: } \\
\text { a. Continuous } \\
\text { pressure and } \\
\text { shear against } \\
\text { the skin* } \\
\text { b. Squeezed } \\
\text { objects falling } \\
\text { on the body } \\
\text { c. The pressure } \\
\text { of clothes } \\
\text { attached to the } \\
\text { body }\end{array}$ & 1 & Retained & 0.53 & $\begin{array}{l}0.54 \\
\text { Cronbach's } \\
\text { Alpha if item } \\
\text { deleted = } 0.81\end{array}$ & $\begin{array}{l}\text { Options: } \\
\begin{array}{l}b=0.13 \\
c=0.34\end{array}\end{array}$ & & $\begin{array}{l}0.67 \\
\text { (factor } \\
2 \text { ) }\end{array}$ \\
\hline \multicolumn{9}{|c|}{ Note: } \\
\hline \multicolumn{9}{|c|}{ * correct answers } \\
\hline \multicolumn{9}{|c|}{ a Value of person correlation (r-value) smaller than r-table } \\
\hline \multicolumn{9}{|c|}{${ }^{b}$ Value of item difficulty smaller than 0.10 or larger than 0.90} \\
\hline
\end{tabular}




\begin{tabular}{|c|c|c|c|c|c|c|c|c|}
\hline 8 & $\begin{array}{l}\text { Pressure injuries } \\
\text { in older adults } \\
\text { can cause: } \\
\text { a. Pain and } \\
\text { infection* } \\
\text { b. Nausea and } \\
\text { vomiting } \\
\text { c. Urinary } \\
\text { incontinence }\end{array}$ & 1 & Retained & 0.67 & $\begin{array}{l}0.62 \\
\text { Cronbach's } \\
\text { Alpha if item } \\
\text { deleted = } 0.81\end{array}$ & $\begin{array}{l}\text { Options: } \\
b=0.14 \\
c=0.19\end{array}$ & Retained & $\begin{array}{l}0.48 \\
\text { (factor } \\
\text { 2) }\end{array}$ \\
\hline 9 & $\begin{array}{l}\text { Pressure injuries } \\
\text { in older adults } \\
\text { can make them: } \\
\text { a. Difficult to } \\
\text { mobile* } \\
\text { b. Difficult to } \\
\text { urinate } \\
\text { c. Difficult to } \\
\text { concentrate }\end{array}$ & $<1$ & Deleted & $\begin{array}{l}\text { Item } \\
\text { deleted/ } \\
\text { not } \\
\text { tested }\end{array}$ & $\begin{array}{l}\text { Item deleted/ } \\
\text { not tested }\end{array}$ & $\begin{array}{l}\text { Item } \\
\text { deleted/ } \\
\text { not tested }\end{array}$ & $\begin{array}{l}\text { Item } \\
\text { deleted/ } \\
\text { not tested }\end{array}$ & $\begin{array}{l}\text { Item } \\
\text { deleted/ } \\
\text { not } \\
\text { tested }\end{array}$ \\
\hline 10 & $\begin{array}{l}\text { What will } \\
\text { happen if } \\
\text { redness in the } \\
\text { skin of older } \\
\text { adults is left } \\
\text { untreated? } \\
\text { a. It will develop } \\
\text { into deep } \\
\text { pressure ulcers* } \\
\text { b. It becomes } \\
\text { blackish and } \\
\text { then heals. } \\
\text { c. It will heal } \\
\text { itself when the } \\
\text { skin is dry. }\end{array}$ & 1 & Retained & $0.08^{b}$ & $\begin{array}{l}\mathbf{0 . 3 6 ^ { \mathbf { c } }} \\
\text { Cronbach's } \\
\text { Alpha if item } \\
\text { deleted = } 0.83\end{array}$ & $\begin{array}{l}\text { Options: } \\
\mathrm{b}=0.32 \\
\mathrm{c}=0.60\end{array}$ & Deleted & $\begin{array}{l}\text { Item } \\
\text { deleted/ } \\
\text { not } \\
\text { tested }\end{array}$ \\
\hline \multicolumn{9}{|c|}{$\begin{array}{l}\text { Topic: Preventive } \\
\text { strategies that family } \\
\text { caregivers can perform } \\
\text { to prevent Pls }\end{array}$} \\
\hline 11 & $\begin{array}{l}\text { What to do to } \\
\text { prevent pressure } \\
\text { injuries in older } \\
\text { adults? } \\
\text { a. Wear loose } \\
\text { clothes } \\
\text { b. Use footwear } \\
\text { when leaving the } \\
\text { house. } \\
\begin{array}{l}\text { c. Prevent } \\
\text { prolonged } \\
\text { pressure on the } \\
\text { skin* }\end{array}\end{array}$ & 1 & Retained & 0.38 & $\begin{array}{l}0.49 \\
\text { Cronbach's } \\
\text { Alpha if item } \\
\text { deleted = } 0.82\end{array}$ & $\begin{array}{l}\text { Options: } \\
\mathrm{a}=0.42 \\
\mathrm{~b}=0.20\end{array}$ & Retained & $\begin{array}{l}0.44 \\
\text { (factor } \\
1)\end{array}$ \\
\hline
\end{tabular}

Note :

* correct answers

${ }^{a}$ Value of person correlation (r-value) smaller than r-table

b Value of item difficulty smaller than 0.10 or larger than 0.90

${ }^{c}$ Value of discriminant index (D value) smaller than 0.40 


\begin{tabular}{|c|c|c|c|c|c|c|c|c|}
\hline 12 & $\begin{array}{l}\text { What to do to } \\
\text { prevent redness } \\
\text { on older adult's } \\
\text { skin? } \\
\text { a. Prevent shear } \\
\text { on the skin } \\
\text { b. Prevent } \\
\text { applying lotion } \\
\text { on the skin } \\
\text { c. Prevent using } \\
\text { hard mattress }\end{array}$ & $<1$ & Deleted & $\begin{array}{l}\text { Item } \\
\text { deleted/ } \\
\text { not } \\
\text { tested }\end{array}$ & $\begin{array}{l}\text { Item deleted/ } \\
\text { not tested }\end{array}$ & $\begin{array}{l}\text { Item } \\
\text { deleted/ } \\
\text { not tested }\end{array}$ & $\begin{array}{l}\text { Item } \\
\text { deleted/ } \\
\text { not tested }\end{array}$ & $\begin{array}{l}\begin{array}{l}\text { Item } \\
\text { deleted/ }\end{array} \\
\text { not } \\
\text { tested }\end{array}$ \\
\hline 13 & $\begin{array}{l}\text { Pressure ulcers } \\
\text { in older adults } \\
\text { can also be } \\
\text { prevented by: } \\
\text { a. Adequate } \\
\text { feeding and } \\
\text { drinking* } \\
\text { b. Sunbathing } \\
\text { c. Prevent stress } \\
\text { on older adults }\end{array}$ & 1 & Retained & 0.66 & $\begin{array}{l}0.62 \\
\text { Cronbach's } \\
\text { Alpha if item } \\
\text { deleted = } 0.81\end{array}$ & $\begin{array}{l}\text { Options: } \\
b=0.13 \\
c=0.21\end{array}$ & Retained & $\begin{array}{l}0.92 \\
\text { (factor } \\
1)\end{array}$ \\
\hline 14 & $\begin{array}{l}\text { For immobile/ } \\
\text { bedridden older } \\
\text { adults, what } \\
\text { should be done } \\
\text { to prevent } \\
\text { pressure } \\
\text { injuries? } \\
\text { a. Mobilization } \\
\text { to the left and } \\
\text { right sleeping } \\
\text { position* } \\
\text { b. Positioning } \\
\text { the older adults } \\
\text { always sleeps on } \\
\text { their back } \\
\text { without any } \\
\text { wedge } \\
\text { c. Let older } \\
\text { adults sleep } \\
\text { without being } \\
\text { disturbed. }\end{array}$ & 1 & Retained & 0.49 & $\begin{array}{l}0.58 \\
\text { Cronbach's } \\
\text { Alpha if item } \\
\text { deleted = } 0.81\end{array}$ & $\begin{array}{l}\text { Options: } \\
b=0.31 \\
c=0.20\end{array}$ & Retained & $\begin{array}{l}0.95 \\
\text { (factor } \\
1)\end{array}$ \\
\hline 15 & $\begin{array}{l}\text { A thing that } \\
\text { should be done } \\
\text { on older persons' } \\
\text { dry skin to avoid } \\
\text { pressure injuries } \\
\text { is: } \\
\text { a. Apply powder } \\
\text { to keep the skin } \\
\text { dry } \\
\text { b. Moisturizes } \\
\text { dry skin* } \\
\text { c. Cover the dry } \\
\text { skin with a } \\
\text { bandage }\end{array}$ & 1 & Retained & 0.53 & $\begin{array}{l}0.60 \\
\text { Cronbach's } \\
\text { Alpha if item } \\
\text { deleted = } 0.81\end{array}$ & $\begin{array}{l}\text { Options: } \\
a=0.23 \\
c=0.24\end{array}$ & Retained & $\begin{array}{l}0.44 \\
\text { (factor } \\
1)\end{array}$ \\
\hline
\end{tabular}

Note :

* correct answers

${ }^{a}$ Value of person correlation (r-value) smaller than r-table

b Value of item difficulty smaller than 0.10 or larger than 0.90

${ }^{c}$ Value of discriminant index (D value) smaller than 0.40 


\begin{tabular}{|c|c|c|c|c|c|c|c|c|}
\hline 16 & $\begin{array}{l}\text { A thing that } \\
\text { should be done } \\
\text { when an older } \\
\text { persons' skin } \\
\text { turns red is: } \\
\text { a. Let it dry itself } \\
\text { b. Release } \\
\text { pressure and } \\
\text { shear* } \\
\text { c. Give betadine } \\
\text { or iodine. }\end{array}$ & 1 & Retained & 0.75 & $\begin{array}{l}0.70 \\
\text { Cronbach's } \\
\text { Alpha if item } \\
\text { deleted = } 0.81\end{array}$ & $\begin{array}{l}\text { Options: } \\
a=0.15 \\
c=0.10\end{array}$ & Retained & $\begin{array}{l}0.45 \\
\text { (factor } \\
1 \text { ) }\end{array}$ \\
\hline 17 & $\begin{array}{l}\text { A thing that } \\
\text { should be done } \\
\text { if the skin of an } \\
\text { older adult } \\
\text { shows deep } \\
\text { pressure injury } \\
\text { is: } \\
\text { a. Take the older } \\
\text { adults to health } \\
\text { care services* } \\
\text { b. Treat using } \\
\text { honey } \\
\text { c. Let it open }\end{array}$ & 1 & Retained & 0.71 & $\begin{array}{l}0.59 \\
\text { Cronbach's } \\
\text { Alpha if item } \\
\text { deleted = } 0.81\end{array}$ & $\begin{array}{l}\text { Options: } \\
\mathrm{b}=0.17 \\
\mathrm{c}=0.12\end{array}$ & Retained & $\begin{array}{l}0.95 \\
\text { (factor } \\
1 \text { ) }\end{array}$ \\
\hline 18 & $\begin{array}{l}\text { What to do to } \\
\text { prevent deep } \\
\text { pressure injuries } \\
\text { in older adults: } \\
\text { a. Consult the } \\
\text { injuries to health } \\
\text { care provider* } \\
\text { b. Apply a } \\
\text { traditional } \\
\text { medicine like } \\
\text { honey or coffee } \\
\text { c. I do not know } \\
\text { the answer }\end{array}$ & $<1$ & Deleted & $\begin{array}{l}\text { Item } \\
\text { deleted/ } \\
\text { not } \\
\text { tested }\end{array}$ & $\begin{array}{l}\text { Item deleted/ } \\
\text { not tested }\end{array}$ & $\begin{array}{l}\text { Item } \\
\text { deleted/ } \\
\text { not tested }\end{array}$ & $\begin{array}{l}\text { Item } \\
\text { deleted/ } \\
\text { not tested }\end{array}$ & $\begin{array}{l}\text { Item } \\
\text { deleted/ } \\
\text { not } \\
\text { tested }\end{array}$ \\
\hline 19 & $\begin{array}{l}\text { Using a special } \\
\text { mattress for } \\
\text { older adults can } \\
\text { prevent pressure } \\
\text { injuries. This } \\
\text { statement is: } \\
\text { a. True* } \\
\text { b. False } \\
\text { c. I do not know }\end{array}$ & 1 & Added & 0.49 & $\begin{array}{l}0.58 \\
\text { Cronbach's } \\
\text { Alpha if item } \\
\text { deleted = } 0.81\end{array}$ & $\begin{array}{l}\text { Options: } \\
\begin{array}{l}b=0.36 \\
c=0.15\end{array}\end{array}$ & Retained & $\begin{array}{l}0.92 \\
\text { (factor } \\
1 \text { ) }\end{array}$ \\
\hline & & & & & & & & \\
\hline & ect answers & & & & & & & \\
\hline & of person corre & 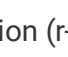 & lue) smal & han r-table & & & & \\
\hline & of item difficult & nal & tan 0.10 & ger than & & & & \\
\hline
\end{tabular}

In the data analysis of phase II, two items (items no.1 and no.10) were identified who had item-total correlation much lower than those of the other items. These two items did not contributed to the Cronbach's alpha; means that if these two items were deleted, the Cronbach's alpha was higher. Item no.10 also had a difficulty index < 0.10. As a result, item no.1 and 10 were removed from the instrument, leaving 12 items with a good difficulty index (mean $=0.57$ ), good discriminating index (mean $=0.59$ ) and good distractors of the multiple-choice alternatives. The dimensionality of all 12 items were tested using Principal Factor Analysis (PFA), generating three factors for the Eigenvalue greater than 1 (as shown in Fig. 2). Factor 1 represented topic about PI prevention (item no.6,7,8,9,10), factor 2 was about cause and consequences of PIs (item no.7 and 8 ), and factor 3 related 
to characteristic of older adults and PI (item no.1,2,3). These three factors together explained $64.4 \%$ of total variance. Factor loading of these 12 items ranged from $0.40-0.92$.

A Confirmatory Factor Analysis with three factor was run for these 12 items in phase III among 240 family caregivers. The results showed that a model of three factor was accepted $(\mathrm{CFI}=0.87 ; \mathrm{TLI}=; 0.82$ and RMSEA $=0.04)$. The internal consistency of Cronbach's alpha was 0.83 . All these results show that the KAP-PI instrument in the knowledge domain with 12 items can be used as an instrument to measure family caregivers' knowledge prevention among community-dwelling older adults.

\section{Attitude domain}

Table 3 shows the items generated in the item development and psychometric evaluation of the items in the attitude domain. At the beginning, ten items (items no.1-10) were generated. Based on the content validity, two (items no.2 and 6) of these items were deleted due to the CVI<1 of each, while one item was added to the supporting interface (item no.11), leaving nine items to be included in phase II. 
Table 3

Attitude domain

\begin{tabular}{|c|c|c|c|c|c|c|c|}
\hline \multirow[t]{2}{*}{ No } & \multirow[t]{2}{*}{ Domain and item generation } & \multirow[t]{2}{*}{ Phase I } & \multicolumn{2}{|l|}{ Phase II } & \multicolumn{3}{|l|}{ Phase III } \\
\hline & & & $\begin{array}{l}\text { PFA (n } \\
=120) \\
\text { Factor } \\
\text { Loading }\end{array}$ & $\begin{array}{l}\text { Reliability } \\
\text { (Cronbach's } \\
\text { Alpha) }\end{array}$ & $\begin{array}{l}\text { CFA (n } \\
=240) \\
\text { Factor } \\
\text { Loading }\end{array}$ & $\begin{array}{l}\text { Reliability } \\
\text { (Cronbach's } \\
\text { Alpha) }\end{array}$ & $\begin{array}{l}\text { "fit indices" } \\
\text { of CFA }\end{array}$ \\
\hline 1. & $\begin{array}{l}\text { I am responsible for the health of the older relative in } \\
\text { my house }\end{array}$ & $\begin{array}{l}\text { Retained } \\
(\mathrm{I}-\mathrm{CVI}= \\
1)\end{array}$ & 0.69 & 0.92 & 0.66 & 0.93 & $\begin{array}{l}\text { Tucker- } \\
\text { Lewis Index/ } \\
\text { TLI = } 0.83\end{array}$ \\
\hline 2. & $\begin{array}{l}\text { I am responsible for pressure injury problems in the } \\
\text { older relative in my house }\end{array}$ & $\begin{array}{l}\text { Deleted } \\
(\mathrm{I}-\mathrm{CVI}< \\
1)\end{array}$ & $\begin{array}{l}\text { Item } \\
\text { deleted/ } \\
\text { not } \\
\text { tested }\end{array}$ & & $\begin{array}{l}\text { Item } \\
\text { deleted/ } \\
\text { not } \\
\text { tested }\end{array}$ & & $\begin{array}{l}\text { Fit Index/ } \\
\text { CFI = 0.87 } \\
\text { RMSEA = } \\
0.02 .\end{array}$ \\
\hline 3. & $\begin{array}{l}\text { The personal hygiene of the older relative in my house } \\
\text { must be cared for carefully }\end{array}$ & $\begin{array}{l}\text { Retained } \\
(\mathrm{I}-\mathrm{CVI}= \\
1)\end{array}$ & 0.71 & & 0.68 & & \\
\hline 4. & $\begin{array}{l}\text { I have to pay attention to the skin moisture and hygiene } \\
\text { of the older relative in my house. }\end{array}$ & $\begin{array}{l}\text { Retained } \\
(\mathrm{I}-\mathrm{CVI}= \\
1)\end{array}$ & 0.79 & & 0.75 & & \\
\hline 5. & $\begin{array}{l}\text { It is important to pay attention to the food and drink of } \\
\text { the older relative in my house }\end{array}$ & $\begin{array}{l}\text { Retained } \\
(\mathrm{I}-\mathrm{CVI}= \\
1)\end{array}$ & 0.88 & & 0.83 & & \\
\hline 6. & $\begin{array}{l}\text { I am responsible for the nutritional problem in the older } \\
\text { relative in my house }\end{array}$ & $\begin{array}{l}\text { Deleted } \\
(\mathrm{I}-\mathrm{CVI}< \\
1)\end{array}$ & $\begin{array}{l}\text { Item } \\
\text { deleted/ } \\
\text { not } \\
\text { tested }\end{array}$ & & $\begin{array}{l}\text { Item } \\
\text { deleted/ } \\
\text { not } \\
\text { tested }\end{array}$ & & \\
\hline 7. & $\begin{array}{l}\text { Pressure injuries on the older relative in my house } \\
\text { should be prevented }\end{array}$ & $\begin{array}{l}\text { Retained } \\
(\mathrm{I}-\mathrm{CVI}= \\
1)\end{array}$ & 0.87 & & 0.86 & & \\
\hline 8. & $\begin{array}{l}\text { Helping the older relative in my house in their activities } \\
\text { and movements is my responsibility }\end{array}$ & $\begin{array}{l}\text { Retained } \\
(\mathrm{I}-\mathrm{CVI}= \\
1)\end{array}$ & 0.79 & & 0.81 & & \\
\hline 9. & $\begin{array}{l}\text { Immobile older relative in my house need to be helped } \\
\text { in movement and positioning }\end{array}$ & $\begin{array}{l}\text { Retained } \\
(\mathrm{I}-\mathrm{CVI}= \\
1)\end{array}$ & 0.77 & & 0.77 & & \\
\hline 10. & $\begin{array}{l}\text { The older relative in my house who experience pressure } \\
\text { injuries need to be checked to health care service }\end{array}$ & $\begin{array}{l}\text { Retained } \\
(\mathrm{I}-\mathrm{CVI}= \\
1)\end{array}$ & 0.80 & & 0.80 & & \\
\hline 11. & $\begin{array}{l}\text { The older relative in my house who are at risk of getting } \\
\text { pressure injuries need a special mattress to prevent } \\
\text { pressure injuries }\end{array}$ & $\begin{array}{l}\text { Added } \\
(\mathrm{I}-\mathrm{CVI}= \\
1)\end{array}$ & 0.78 & & 0.75 & & \\
\hline
\end{tabular}

A Principal Factor Analysis (PFA) with oblique rotation was run for phase II. All requirements for performing PFA were met (KMO MSA = 0.89; Bartletts Test of sphericity (Sig.) $=0.00)$. All nine items tested had factor loading $>0.40$ of each $(0.64-0.87)$, which means that no items had to be deleted. As shown in Figure. 3, these nine items constructed one factor for the Eigenvalue greater than 1 and explained $62.13 \%$ of the total variability, which is higher than the required $60 \%(44))$.

In phase III, all nine items were tested among 240 family caregivers. A Confirmatory Factor Analysis was run resulting in "fit indices" including Tucker-Lewis Index/ TLI of 0.83; Comparative Fit Index/ CFI of 0.87; and RMSEA of 0.02. These three "fit indices" indicated that the unidimensional model which resulted from the PCA in phase II, was confirmed as the model fit. The internal consistency of the final versions of the KAP-PI instrument in the attitude domain had a Cronbach's alpha of 0.93 , indicating high reliability. In conclusion, all validity and reliability test results indicate that the nine items can be trusted as a means of measuring family caregivers' attitudes toward pressure injury prevention in communitydwelling older adults. 


\section{Practice domain}

Table 4 shows the items generated in the practice domain and its psychometric evaluation. Four topics and 13 items (items no.1-13) were developed in phase I. Of the 13 items, one item (item no.13) was deleted were deleted due to the $\mathrm{CVI}<1$ of each, while one item was added to the supporting interface (item no.14), leaving 13 items to be tested for phase II. A PFA with oblique rotation was run in phase II. All requirements for performing PFA were met (KMO MSA = 0.85; Bartletts Test of sphericity (Sig.) $=0.00)$. When looking to the factor loading of each item, item no.12 had a factor loading $<0.40(0.14)$, indicating the item should be deleted from the instrument. After deleting item no.12, the PFA with oblique rotation was re-run and this second PFA resulted in a factor loading $>0.40$ of each item (0.41-0.82). As shown in Fig. 4 , all 12 items constructed three factors for the Eigenvalue greater than 1, i.e. factor 1 (item no. 6, 7, 8,and 9), factor 2 (item no.1, 2, 10, 11, and 12), and factor 3 (item no. 3, 4, and 5). These three factors explained $66.11 \%$ of the total variability (required not less than $60 \%(44)$ ). 
Table 4

Practice domain

\begin{tabular}{|c|c|c|c|c|c|c|c|c|c|}
\hline \multirow[t]{4}{*}{ No } & \multirow{4}{*}{$\begin{array}{l}\text { Domain and item } \\
\text { generation }\end{array}$} & \multirow[t]{4}{*}{ Phase I } & \multicolumn{4}{|c|}{ Phase II } & \multicolumn{3}{|c|}{ Phase III } \\
\hline & & & \multicolumn{3}{|c|}{ PFA $(n=120)$} & \multirow{3}{*}{$\begin{array}{l}\text { Reliability } \\
\text { (Cronbach's } \\
\text { Alpha) }\end{array}$} & CFA $(n=$ & 240) & \\
\hline & & & \multicolumn{3}{|c|}{ Factor Loading } & & \multicolumn{3}{|c|}{ Factor Loading } \\
\hline & & & $\begin{array}{l}\text { Factor } \\
1\end{array}$ & $\begin{array}{l}\text { Factor } \\
2\end{array}$ & $\begin{array}{l}\text { Factor } \\
3\end{array}$ & & $\begin{array}{l}\text { Factor } \\
1\end{array}$ & $\begin{array}{l}\text { Factor } \\
2\end{array}$ & $\begin{array}{l}\text { Factor } \\
3\end{array}$ \\
\hline & \multirow{2}{*}{\multicolumn{2}{|c|}{$\begin{array}{l}\text { Topic: Activities performed to } \\
\text { support older adults to meet } \\
\text { their nutritional and fluid needs }\end{array}$}} & & & & 0.87 & & & \\
\hline & & & & & & & & & \\
\hline 1. & $\begin{array}{l}\text { Provide healthy } \\
\text { food for the older } \\
\text { relative in my house }\end{array}$ & $\begin{array}{l}\text { Retained } \\
(I-C V I= \\
1)\end{array}$ & & 0.74 & & & & 0.51 & \\
\hline \multirow[t]{3}{*}{2.} & $\begin{array}{l}\text { Provide mineral } \\
\text { water for the older } \\
\text { relative in my house } \\
\text { at least } 8 \text { glasses in } \\
\text { a day }\end{array}$ & $\begin{array}{l}\text { Retained } \\
(\mathrm{I}-\mathrm{CVI}= \\
1)\end{array}$ & & 0.53 & & & & 0.55 & \\
\hline & \multirow{2}{*}{\multicolumn{5}{|c|}{$\begin{array}{l}\text { Topic: Activities performed to } \\
\text { support older adults in } \\
\text { mobilization and repositioning }\end{array}$}} & & & & \\
\hline & & & & & & & & & \\
\hline 3. & $\begin{array}{l}\text { Helping the older } \\
\text { relative in my house } \\
\text { to do activities if } \\
\text { they cannot do it } \\
\text { him/herself }\end{array}$ & $\begin{array}{l}\text { Retained } \\
(\mathrm{I}-\mathrm{CVI}= \\
1)\end{array}$ & & & 0.86 & & & & 0.87 \\
\hline 4. & $\begin{array}{l}\text { Helping for the older } \\
\text { relative in my house } \\
\text { to move if they } \\
\text { cannot do it } \\
\text { him/herself }\end{array}$ & $\begin{array}{l}\text { Retained } \\
(I-C V I= \\
1)\end{array}$ & & & 0.78 & & & & 0.82 \\
\hline \multirow[t]{3}{*}{5.} & $\begin{array}{l}\text { Helping the } \\
\text { bedridden older } \\
\text { relative in my house } \\
\text { to change their } \\
\text { position } \\
\text { (positioning) } \\
\text { regularly if they } \\
\text { cannot do it } \\
\text { him/herself }\end{array}$ & $\begin{array}{l}\text { Retained } \\
(I-C V I= \\
1)\end{array}$ & & & 0.61 & & & & 0.87 \\
\hline & \multirow{2}{*}{\multicolumn{5}{|c|}{$\begin{array}{l}\text { Topic: Activities performed to } \\
\text { support older adults in skin } \\
\text { hygiene and moisture care }\end{array}$}} & & & & \\
\hline & & & & & & & & & \\
\hline 6. & $\begin{array}{l}\text { Prevent the older } \\
\text { relative in my house } \\
\text { from using damp } \\
\text { and wet clothes, } \\
\text { including changing } \\
\text { diapers regularly (if } \\
\text { they use diapers) }\end{array}$ & $\begin{array}{l}\text { Retained } \\
(I-C V I= \\
1)\end{array}$ & 0.75 & & & & 0.64 & & \\
\hline 7. & $\begin{array}{l}\text { Prevent long } \\
\text { pressure on the } \\
\text { body of the older } \\
\text { relative in my house }\end{array}$ & $\begin{array}{l}\text { Retained } \\
(I-C V I= \\
1)\end{array}$ & 0.85 & & & & 0.87 & & \\
\hline 8. & $\begin{array}{l}\text { Moisturizing the } \\
\text { skin of the older } \\
\text { relative in my house } \\
\text { by giving } \\
\text { lotions/oils }\end{array}$ & $\begin{array}{l}\text { Retained } \\
(I-C V I= \\
1)\end{array}$ & 0.76 & & & & 0.85 & & \\
\hline
\end{tabular}




\begin{tabular}{|c|c|c|c|c|}
\hline 9. & $\begin{array}{l}\text { Check the entire } \\
\text { skin of the older } \\
\text { relative in my house } \\
\text { for redness }\end{array}$ & $\begin{array}{l}\text { Retained } \\
(\mathrm{I}-\mathrm{CVI}= \\
1)\end{array}$ & 0.71 & 0.67 \\
\hline & \multicolumn{3}{|l|}{$\begin{array}{l}\text { Topic: Activities } \\
\text { performed to } \\
\text { support older adults } \\
\text { to maintain their } \\
\text { health and ensure } \\
\text { access to health } \\
\text { care services }\end{array}$} & \\
\hline 10. & $\begin{array}{l}\text { Maintain the } \\
\text { environmental } \\
\text { hygiene of for the } \\
\text { older relative in my } \\
\text { house }\end{array}$ & $\begin{array}{l}\text { Retained } \\
(I-C V I= \\
1)\end{array}$ & 0.68 & 0.67 \\
\hline 11. & $\begin{array}{l}\text { Took the older } \\
\text { relative in my house } \\
\text { to health services if } \\
\text { they suffer from } \\
\text { wounds }\end{array}$ & $\begin{array}{l}\text { Retained } \\
(\mathrm{I}-\mathrm{CVI}= \\
1)\end{array}$ & 0.61 & 0.74 \\
\hline 12. & $\begin{array}{l}\text { Took the older adult } \\
\text { to health services if } \\
\text { they had a health } \\
\text { problem }\end{array}$ & $\begin{array}{l}\text { Retained } \\
(\mathrm{I}-\mathrm{CVI}= \\
1)\end{array}$ & $\begin{array}{l}\text { MSA }=0.45(<0.50) \\
\text { Item was not included in } \\
\text { PCA. }\end{array}$ & $\begin{array}{l}\text { Item deleted/ } \\
\text { not tested }\end{array}$ \\
\hline \multirow[t]{2}{*}{13.} & $\begin{array}{l}\text { Contact health care } \\
\text { provider to get their } \\
\text { suggestion } \\
\text { according to } \\
\text { condition of the } \\
\text { older relative }\end{array}$ & $\begin{array}{l}\text { Deleted } \\
(\mid-C V I< \\
1)\end{array}$ & $\begin{array}{l}\text { Item deleted/ } \\
\text { not tested }\end{array}$ & $\begin{array}{l}\text { Item deleted/ } \\
\text { not tested }\end{array}$ \\
\hline & \multicolumn{4}{|c|}{$\begin{array}{l}\text { Topic: Support older adult to } \\
\text { have a special mattress }\end{array}$} \\
\hline 14. & $\begin{array}{l}\text { Provide a special } \\
\text { mattress for a } \\
\text { bedridden elderly } \\
\text { relative in my house }\end{array}$ & $\begin{array}{l}\text { Added }(1- \\
\mathrm{CVI}=1)\end{array}$ & 0.64 & 0.49 \\
\hline
\end{tabular}

In phase III, all 12 items were tested among 240 family caregivers. A Confirmatory Factor Analysis was run to check whether the three factors resulted by Principal Factor Analysis (PFA) was a good model or not. The CFA resulted in "fit indices", i.e. Tucker-Lewis Index/ TLI of 0.83; Comparative Fit Index/ CFI of 0.87; and RMSEA of 0.06. These three "fit indices" indicated that the three factor model resulting from the PCA in phase II, was confirmed as the model fit for practice domain. The correlations between factors were $>0.60$. The internal consistency a Cronbach's alpha of factor 1,2 and 3 were $0.80,0.73$, and 0.87 respectively. The overall internal consistency of the final version of the KAP-PI instrument in the practice domain had a Cronbach's alpha of 0.89 , indicating high reliability. In conclusion, all validity and reliability test results indicate that the 12 items can be trusted as a means of measuring family caregivers' practice toward pressure injury prevention in community-dwelling older adults.

\section{Discussion}

In this study, following a guideline described by Boateng et al. (27), an instrument to measure knowledge, attitude, and practice of family caregivers to prevent PIs among community-dwelling older adults (KAP-PI) in Indonesia was developed and psychometrically tested. The results demonstrate that the final version of the KAP-PI was valid and had Chronbach's Alpha values of $0.83,0.93$ and 0.89 in the respective knowledge, attitude and practice domains. This also indicates high reliability.

Background characteristics of the sample show heterogeneity with regard to gender, age group, educational level, employment and relationship to the community-dwelling older adult. This sample is a good representation of the targeted population of family caregivers in Indonesia who mostly care for their parents or relative at home (extended family). Irrespective of the fact that the group was highly heterogeneous, the KAP-PI instrument showed good performance in the statistic analysis (33).

In the final version of the KAP-PI instrument, the knowledge domain consists of 12 items. To assess the validity of the items, item difficulty, discriminating index, and quality of response alternatives were measured. These measurements are commonly used in studies focused on developing knowledge questionnaires, such as described by Beeckman et al. (2010) and Manderlier et al. (2017), who developed an instrument to measure nurse's knowledge about PI prevention that had been used in many studies (45-48). Even though our target group, family caregivers, is different from those described in these studies (nurses), it is important that family caregivers know definition, symptoms, and consequences of Pls 
prior to knowledge about activities needed to prevent PI $(21,30)$. These topics were not covered in a prior study by Bellon and Pancarbo $(26)$, who also developed and tested a questionnaire to assess family caregiver's knowledge about PI prevention. Additional topics about definition, symptoms, and consequences of PIs were included in the KAP-PI instrument. However, study by Bellon and Pancarbo and our study covered almost the same points of knowledge about activities of PI prevention. Furthermore, construct evaluation of the knowledge domain of the KAP-PI instrument generated three factors. Hence, when using the KAP-PI instrument in a real survey, the results should be analyzed for each factor.

When looking at the attitude domain, the nine items are highly correlated, constructing the unidimensionality of the attitude domain of the KAP-PI instrument. Attitude is an affective aspect of a person that causes him or her to take a certain action (49). Measuring attitude is essential if a specific behavior or practice is an outcome of the intervention; for example, when looking at health education as an intervention you hope to see different behavior afterwards $(25,50)$. In this study, the attitude domain reflects the beliefs and values of family caregivers towards PI prevention. The most important aspect of attitude is the willingness of family caregivers to support older adults in preventing Pls. The willingness to help others is an essential factor for family caregivers and any informal caregivers before being involved in an education or empowerment program (51-54). In family nursing practice, affection is a binding domain that should be considered when planning and performing a family nursing intervention (29). Several studies have developed instruments in affective or emotional domains to assess family functioning among patients with different conditions. For example, the Iceland-Expressive Family Functioning Questionnaire (ICE-EFFQ) measuring expressive family functioning when experiencing acute or chronic illnesses (55). The ICE-EFFQ was psychometrically tested using the same data analysis techniques (EFA followed by CFA) as used in our study. The KAP-PI instrument measures the affective aspect of family caregivers in PI prevention. This current study added inventory family affective assessment tools to prior published instruments such as Family functioning, Health, and Social Support (FAFHES) used for a family of an adult cardiac patient (56) and Family Assessment Device (FAD) measuring family functioning in general (57, 58).

In the practice domain, three topics emerged and were validated: basic support, reposition and mobilization support, and skin hygiene and moisture support. The Likert scale was used to assess family caregiver activities about PI prevention for their older relatives. Nurses working with families, which in Indonesia usually performed by community nurses, can use the KAP-PI instrument to get insight to what extent family caregivers actually perform the essential activities to prevent PIs in their relatives. Nurses should consider the nature of family caregivers' support for their older relatives (59). By assessing family caregiver's knowledge, attitude, and practice using the KAP-PI instrument, nurses can arrange a training program to increase families' competencies in their authority as informal caregivers to prevent PI in their older relatives.

\section{Limitations}

One limitation of this study could be that the content validity was based on only three experts. To account for the limited number of included experts, only the items rated quite relevant or highly relevant by all experts were retained in the instrument. Also, a thorough statistical analysis was done in phase 2 and 3 to ensure validity and reliability of the instrument. Another limitation is that test-retest reliability was not determined. However, validity and reliability were derived from two independent samples from two different data collection procedures, including a relatively large sample size (120 participants in phase II and 240 participants in phase III). Therefore, we believe the instrument was thoroughly developed and is good to use among our intended population.

\section{Conclusion}

An instrument to assess knowledge, attitude and practice of Family Caregiver on Preventing Pressure Injuries (KAP-PI) among community-dwelling older adults in Indonesia was developed and validated. A 12-item knowledge domain, a 9-item attitude domain, and a 12-item practice domain were designed based on a guided construction process. The validity and reliability of the instrument were statistically acceptable. The instrument can be used in family nursing practice, education, and research to assess the function of family caregivers in preventing pressure injuries among community-dwelling older people in Indonesia.

\section{Abbreviations}

CFA : Confirmatory Factor Analysis

KAP_PI : Knowledge, Attitude and Practice of family caregivers to prevent PIs among community-dwelling older adults in Indonesia

I-CVI : Item content validity index

NPUAP : National Pressure Ulcer Advisory Panel

PFA : Principal Factor Analysis

$\mathrm{PI}$ : Pressure Injury

PPPIA : Pan Pacific Pressure Injury Alliance 
S-CVI : Instrument content validity index

UPUAP : European Pressure Ulcer Advisory Panel

\section{Declarations}

\section{Ethics approval and consent to participate}

Two governmental institutions that have responsibilities in health care and community protection approved the research project before undertaken (the Indonesian Health Care Agency \#070/13472-Dinkes and the National Unity Agency, Politics and Protection of the Regional People \#070/3177/Bakesbangpol). Participants received information about the study and signed consent if they agreed to participate. Participants were not obligated to participate and could refuse participation before and during the data collection.

\section{Consent for publication}

Not applicable

\section{Availability of data and materials}

All data generated or analysed during this study are included in this published article [and its supplementary information file].

\section{Competing interests}

The authors declare that they have no competing interests

\section{Funding}

This study was funded by the Indonesia Endowment Fund for Education (LPDP) and the Ministry of Research, Technology and Higher Education of the Republic of Indonesia (BUDI-LN Scholarship).

The funding for publication was supported by Maastricht University.

\section{Authors' contributions}

All authors made substantial contributions to the conception, study design, analysis and interpretation of data. SPS was responsible for the acquisition of the data and drafted the first manuscript with the supervision of IE, JS, CL, RH, YA and DB. SPS and EAS contributed to the data collection. All authors, SPS, IE, JS, RH, CL, YA, EAS, and DB, revised the manuscript critically for important intellectual content. Finally, all authors read and approved the final manuscript.

\section{Acknowledgements}

We would like to thank all participating municipalities in Bandung and Departement of Health Bandung City, nurses and family caregivers who participated actively in this study. Our thanks also go to the LPZ International research group and the Maastricht, Graz, and Bern doctoral nursing science participants.

\section{References}

1. European Pressure Ulcer Advisory Panel NPIAPaPPPIA. Prevention and Treatment of Pressure Ulcers/ Injuries: Clinical Practice Guide: EPUAP/NPIAP/PPPIA; 2019. Available from: https://www.epuap.org/pu-guidelines/.

2. Corbett LQ, Funk M, Fortunato G, O'Sullivan DM. Pressure Injury in a Community Population: A Descriptive Study. J Wound Ostomy Continence Nurs. 2017;44(3):221-7.

3. Bogaisky M, Dezieck L. Early Hospital Readmission of Nursing Home Residents and Community-Dwelling Elderly Adults Discharged from the Geriatrics Service of an Urban Teaching Hospital: Patterns and Risk Factors. Journal of the American Geriatrics Society. 2015;63(3):548-52.

4. Stevenson R, Collinson M, Henderson V, Wilson L, Dealey C, McGinnis E, et al. The prevalence of pressure ulcers in community settings: an observational study. Int J Nurs Stud. 2013;50(11):1550-7. 
5. Sari SP, Everink IH, Sari EA, Afriandi I, Amir Y, Lohrmann C, et al. The prevalence of pressure ulcers in community-dwelling older adults: A study in an Indonesian city. International Wound Journal. 2019;16(2):534-41.

6. World Population [Internet]. 2021.

7. UN Data: A world of Information [Internet]. 2021 [cited 19 January 2021].

8. Indonesia BPS. Statistik Penduduk Lanjut Usia 2019. @Badan Pusat Statistik; 2020.

9. Arifin EN, Braun KL, Hogervorst E. Three pillars of active ageing in Indonesia. Asian Population Studies. 2012;8(2):207-30.

10. Niehof A. Ageing and the elderly in Indonesia; Identifying key issues. Bijdragen tot de taal-, land-en volkenkunde/Journal of the Humanities and Social Sciences of Southeast Asia. 1995;151(3):422 - 37.

11. Amir Y, Tan FE, Halfens R, Lohrmann C, Schols J. Pressure Ulcer Prevalence and Care in Indonesian Hospitals: A Multicenter, Cross-sectional Evaluation Using an Extended Donabedian Model. Ostomy Wound Manage. 2017;63(2):8-23.

12. Amir Y, Lohrmann C, Halfens RJ, Schols JM. Pressure ulcers in four Indonesian hospitals: prevalence, patient characteristics, ulcer characteristics, prevention and treatment. Int Wound J. 2017;14(1):184-93.

13. Coe NB, Guo J, Konetzka RT, Van Houtven $\mathrm{CH}$. What is the marginal benefit of payment-induced family care? Impact on Medicaid spending and health of care recipients. Health Economics. 2019;28(5):678-92.

14. Eisenberger A, Zeleznik J. Pressure ulcer prevention and treatment in hospices: a qualitative analysis. Journal of Palliative Care. 2003;19(1):914.

15. Martins Pereira S, Maria Soares HI. Pressure ulcers: relatives' perceptions of emotional impact and non-material costs. Revista de Enfermagem Referência. 2012(7):139-48.

16. Nuriyanto A, Rahayuwati L. Family Nursing as an Improvement Strategy of Family Health Index in Indonesia: A Literature Review. Asian Community Health Nursing Research. 2019;1(3):7-16.

17. Tavares BC, Matos E, Cristina I, Maliska A, Benedet SA, Salum NC. Profile Of Patients Diagnosed With Chronic Ulcer Of Diverse Etiology Admitted To An Emergency Unit. Ciência, Cuidado e Saúde. 2016;15(4):624.

18. Oot-Giromini BA. Pressure ulcer prevalence, incidence and associated risk factors in the community. Decubitus. 1993;6(5):24-32.

19. Fateme K, Fariba Y, Aliakbar K, Hassan A. Investigating the Effect of Home-Based Training for Family Caregivers on the Incidence of Bedsore in Patients with Stroke in Ali Ebne Abitaleb Hospital, Zahedan, Iran: A Clinical Trial Study. Medical-Surgical Nursing Journal. 2018;7(3):1-6.

20. Borsting TE, Tvedt CR, Skogestad IJ, Granheim TI, Gay CL, Lerdal A. Prevalence of pressure ulcer and associated risk factors in middle- and older-aged medical inpatients in Norway. J Clin Nurs. 2018;27(3-4):e535-e43.

21. Jan McCulloch B. Family Health Care Nursing: Theory, Practice and Research. Journal of Family Theory \& Review. 2012;4(1):67-70.

22. Suleiman M, Sahal N, Sodemann M, Elsony A, Aro AR. Tuberculosis awareness in Gezira, Sudan: knowledge, attitude and practice case-control survey. EMHJ-Eastern Mediterranean Health Journal 2014; 20 ( 2): 120-129. 2014.

23. Khalil A, Abdalrahim M. Knowledge, attitudes, and practices towards prevention and early detection of chronic kidney disease. International nursing review. 2014;61(2):237-45.

24. Wan TT, Rav-Marathe K, Marathe S. A SYSTEMATIC REVIEW OF KAP-O FRAMEWORK FOR DIABETES. Medical Research Archives. 2016;3(9).

25. Badran IG. Knowledge, attitude and practice the three pillars of excellence and wisdom: a place in the medical profession. Eastern Mediterranean Health Journal. 1995;1(1):8-16.

26. Bellón JA, Pancorbo-Hidalgo PL. family caregivers about prevention of pressure ulcer and dependence-related skin lesions: development and validation. Gerokomos. 2016;27(2):73-9.

27. Boateng GO, Neilands TB, Frongillo EA, Melgar-Quiñonez HR, Young SL. Best Practices for Developing and Validating Scales for Health, Social, and Behavioral Research: A Primer. Frontiers in Public Health. 2018;6(149).

28. National Pressure Ulcer Advisory Panel EPUAP, Pan Pacific Pressure Injury Alliance. Prevention and Treatment of Pressure Ulcers: Quick Reference Guide. E H, editor. Perth: Cambridge Media; 2014.

29. Wright LM, Leahey M. Nurses and families: a guide to family assessment and intervention. 5th ed. Philadelphia: F.A. Davis; 2009.

30. Friedman MM, Bowden VR, Jones E. Family nursing: Research, theory \& practice: Prentice Hall Upper Saddle River, NJ; 2003.

31. Friedman MM. Family nursing: theory \& assessment. New York: Appleton-Century-Crofts; 1981.

32. Bourke J, Kirby A, Doran J. SURVEY \& QUESTIONNAIRE DESIGN: Collecting Primary Data to Answer Research Questions. Ireland: NuBooks; 2016.

33. Haladyna TM. Developing and Validating Multiple-choice Test Items. Mahwah, N.J.: Routledge; 2004.

34. Peer Wv, Hakemulder Jm, Zyngier S. Scientific Methods for the Humanities. Amsterdam, Netherlands: John Benjamins Publishing Company; 2012.

35. Polit DF, Beck CT, Owen SV. Is the CVI an acceptable indicator of content validity? Appraisal and recommendations. Res Nurs Health. 2007;30(4):459-67. 
36. Indonesia PNR. Peraturan Menteri Kesehatan Republik Indonesia Nomor 67 Tahun 2015 Tentang Penyelenggaraan Pelayanan Kesehatan Lanjut Usia. 2015.

37. Taherdoost H. Validity and Reliability of the Research Instrument; How to Test the Validation of a Questionnaire/Survey in a Research. SSRN Electronic Journal. 2016.

38. lantovics LB, Rotar C, Morar F. Survey on establishing the optimal number of factors in exploratory factor analysis applied to data mining. WIREs Data Mining and Knowledge Discovery. 2019;9(2):e1294.

39. Thompson B. Exploratory and confirmatory factor analysis: understanding concepts and applications. Washington, DC: American Psychological Association; 2004. Available from: http://search.ebscohost.com/direct.asp?db=pzh\&jid=\%22200413115\%22\&scope=site

40. Rosseel Y. lavaan: An R Package for Structural Equation Modeling. 2012. 2012;48(2):36.

41. Comprehensive R Archive Network (CRAN).

42. Thompson B. Confirmatory factor analysis decision sequence. Exploratory and confirmatory factor analysis: Understanding concepts and applications. Washington, DC: American Psychological Association; 2004. p. 109-32.

43. Dr. Joyce Fitzpatrick PMBARNFF. Encyclopedia of Nursing Research. New York: Springer Publishing Company; 2018.

44. Holland SM. Principal components analysis (PCA). Department of Geology, University of Georgia, Athens, GA. 2008:30602-2501.

45. Beeckman D, Vanderwee K, Demarré L, Paquay L, Van Hecke A, Defloor T. Pressure ulcer prevention: Development and psychometric validation of a knowledge assessment instrument. International Journal of Nursing Studies. 2010;47(4):399-410.

46. Manderlier B, Van Damme N, Vanderwee K, Verhaeghe S, Van Hecke A, Beeckman D. Development and psychometric validation of PUKAT 2.0, a knowledge assessment tool for pressure ulcer prevention. International Wound Journal. 2017;14(6):1041-51.

47. De Meyer D, Verhaeghe S, Van Hecke A, Beeckman D. Knowledge of nurses and nursing assistants about pressure ulcer prevention: A survey in 16 Belgian hospitals using the PUKAT 2.0 tool. Journal of Tissue Viability. 2019;28(2):59-69.

48. Sari SP, Everink IHJ, Amir Y, Lohrmann C, Halfens RJG, Moore Z, et al. Knowledge and Attitude of Community Nurses on Pressure Injury Prevention: A Cross-sectional Study in an Indonesian City. International Wound Journal. 2021.

49. Stephen WL, Karen AF. Encyclopedia of Communication Theory. Thousand Oaks, Calif: SAGE Publications, Inc; 2009.

50. World Health Organization. Regional Office for the Eastern M. Health education: theoretical concepts, effective strategies and core competencies: a foundation document to guide capacity development of health educators. 2012.

51. Lukman M, Ibrahim K, Yani DI, Sari SP, Juniarti N. Exploring strategies to improve the performance of community health volunteers for tuberculosis care and prevention: A qualitative study. International Journal of Community Based Nursing and Midwifery. 2019;7(4):270-8.

52. Chang Y-P, Schneider JK, Sessanna L. Decisional conflict among Chinese family caregivers regarding nursing home placement of older adults with dementia. Journal of Aging Studies. 2011;25(4):436-44.

53. Miriam B, Jim O, Judith, Phillips, Chris P. Family and Community Life of Older People: Social Networks and Social Support in Three Urban Areas. London: Routledge; 2001.

54. Esandi N, Nolan M, Canga-Armayor N, Pardavila-Belio MI, Canga-Armayor A. Family Dynamics and the Alzheimer's Disease Experience. Journal of Family Nursing. 2021;27(2):124-35.

55. Sveinbjarnardottir EK, Svavarsdottir EK, Hrafnkelsson B. Psychometric Development of the Iceland-Expressive Family Functioning Questionnaire (ICE-EFFQ). Journal of Family Nursing. 2012;18(3):353-77.

56. Åstedt-Kurki P, Tarkka M-T, Rikala M-R, Lehti K, Paavilainen E. Further testing of a family nursing instrument (FAFHES). International Journal of Nursing Studies. 2009;46(3):350-9.

57. Ridenour TA, Daley JG, Reich W. Factor analyses of the family assessment device. Family process. 1999;38(4):497-510.

58. Duckett S. Family assessment device. Archives of physical medicine and rehabilitation. 1997;78(2):234.

59. Hagedoorn El, Paans W, Jaarsma T, Keers JC, van der Schans C, Luttik ML. Aspects of family caregiving as addressed in planned discussions between nurses, patients with chronic diseases and family caregivers: a qualitative content analysis. BMC Nursing. $2017 ; 16(1): 37$.

\section{Figures}




\begin{tabular}{|c|c|c|c|c|c|c|}
\hline \multirow{4}{*}{ 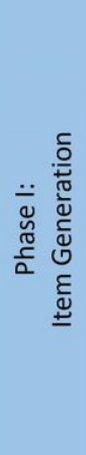 } & & \multicolumn{4}{|c|}{$\begin{array}{l}\text { Theory driven and item generation of } \\
\qquad \text { KAP-PI }\end{array}$} & \\
\hline & \multicolumn{6}{|c|}{$\begin{array}{l}\text { Knowledge domain: four topics, } 18 \text { items } \\
\text { Attitude domain: } 10 \text { items }\end{array}$} \\
\hline & & & \multicolumn{3}{|c|}{ Content and face validity } & \\
\hline & \multicolumn{6}{|c|}{$\begin{array}{l}\text { Preliminary KAP-PI Instrument } \\
\text { Knowledge domain: four topics, } 14 \text { items } \\
\text { Attitude domain: } 9 \text { items } \\
\text { Practice domain: five topics, } 13 \text { items }\end{array}$} \\
\hline \multirow{5}{*}{ 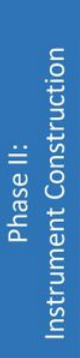 } & & & \multicolumn{3}{|c|}{$\begin{array}{c}\text { Data driven of preliminary of KAP-PI: } \\
\text { survey administration to } 120 \text { of family } \\
\text { caregivers }\end{array}$} & \\
\hline & Domain & Analyses & Item tested & Item reduction & Factor extraction & $\begin{array}{l}\text { Cronbach's } \\
\text { Alpha }\end{array}$ \\
\hline & Knowledge & $\begin{array}{l}\text { Pearson Correlation } \\
\text { Product Moment }\end{array}$ & 14 items & Deleted 2 items & $\begin{array}{l}1 \text { factor } \\
\text { extracted }\end{array}$ & 0.82 \\
\hline & Attitude & $\begin{array}{c}\text { Principal Component } \\
\text { Analyses (PCA) }\end{array}$ & 9 item & $\begin{array}{l}\text { No item } \\
\text { deleted }\end{array}$ & $\begin{array}{c}1 \text { factor } \\
\text { extracted }\end{array}$ & 0.92 \\
\hline & Practice & $\begin{array}{l}\text { Principal Component } \\
\text { Analyses (PCA) }\end{array}$ & 13 items & Deleted 1 item & $\begin{array}{l}3 \text { factors } \\
\text { extracted }\end{array}$ & 0.87 \\
\hline \multirow{5}{*}{ 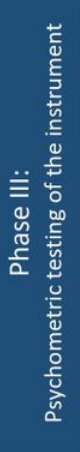 } & & & \multicolumn{3}{|c|}{$\begin{array}{l}\text { Data driven of pre final of KAP-PI: } \\
\text { survey administration to } 240 \text { of family } \\
\text { caregivers }\end{array}$} & \\
\hline & Domain & Analyses & Item tested & $\begin{array}{c}\text { Tests of } \\
\text { dimensionality }\end{array}$ & Test of validity & $\begin{array}{l}\text { Cronbach's } \\
\text { Alpha }\end{array}$ \\
\hline & Knowledge & $\begin{array}{l}\text { CFA and Cronbach's } \\
\text { Alpha }\end{array}$ & 12 items & $\begin{array}{l}\text { Multivocal } \\
\text { model } 3 \\
\text { factors } \\
\text { confirmed }\end{array}$ & $\begin{array}{l}\text { The } 12 \text { items } \\
\text { were valid }\end{array}$ & $\begin{array}{l}0.83 \\
F 1: 0.77 \\
F 2: 0.78 \\
F 3: 0.74\end{array}$ \\
\hline & Attitude & $\begin{array}{l}\text { CFA and Cronbach's } \\
\text { Alpha }\end{array}$ & 9 item & $\begin{array}{l}\text { Univocal model } \\
\quad(1 \text { factor } \\
\text { confirmed })\end{array}$ & $\begin{array}{l}\text { The } 9 \text { items } \\
\text { were valid }\end{array}$ & 0.93 \\
\hline & Practice & $\begin{array}{l}\text { CFA and Cronbach's } \\
\text { Alpha }\end{array}$ & 12 items & $\begin{array}{l}\text { Multivocal } \\
\text { model }(3 \\
\text { factors } \\
\text { confirmed }\end{array}$ & $\begin{array}{l}\text { The } 12 \text { items } \\
\text { were valid }\end{array}$ & $\begin{array}{c}0.89 \\
F 1: 0.80 \\
F 2: 0.73 \\
F 3: 0.87 \\
\end{array}$ \\
\hline
\end{tabular}

\section{Figure 1}

The process of developing and psychometric evaluation of KAPI-PI instrument 


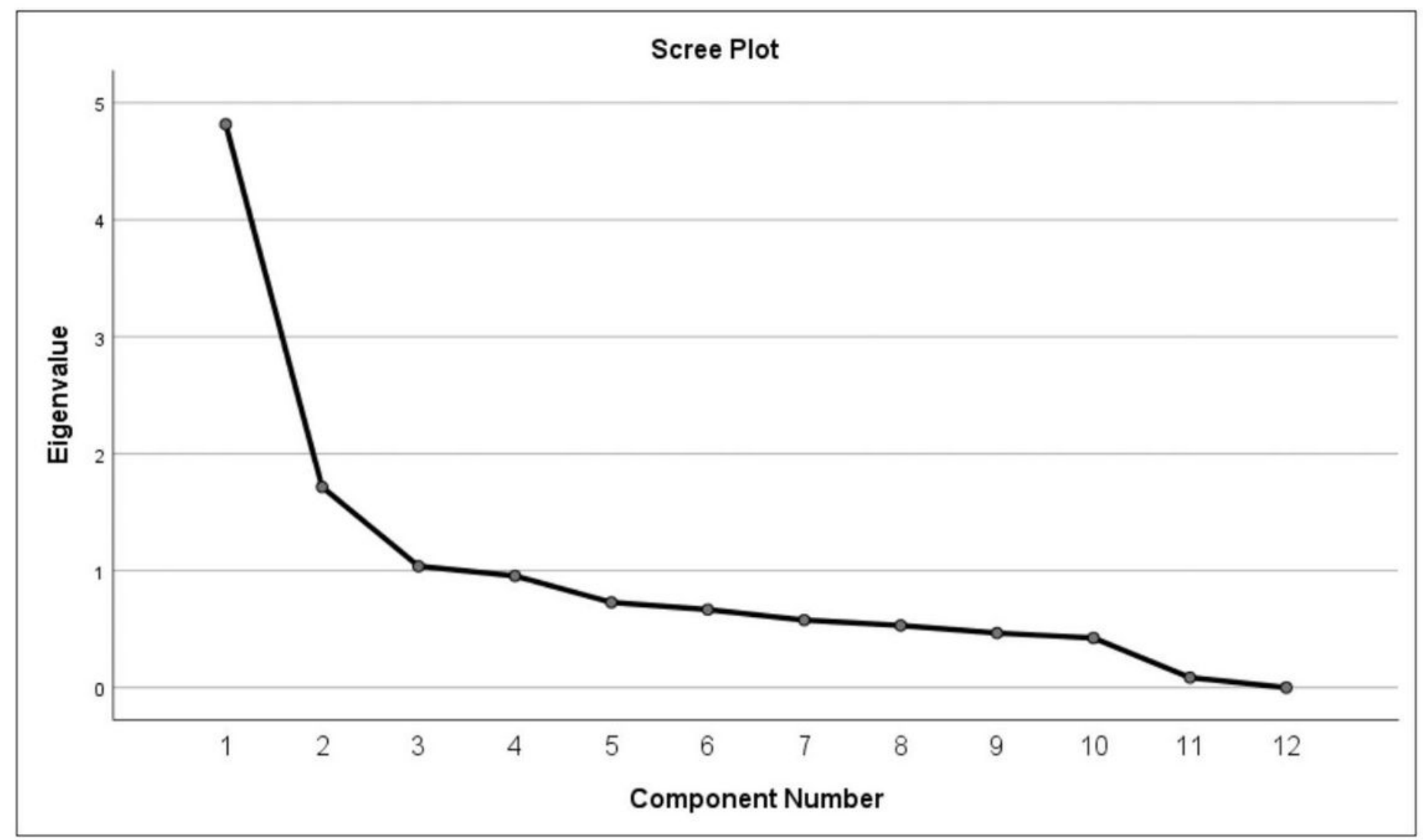

Figure 2

Scree plot resulted from principal factor analysis (PFA) in knowledge domain 


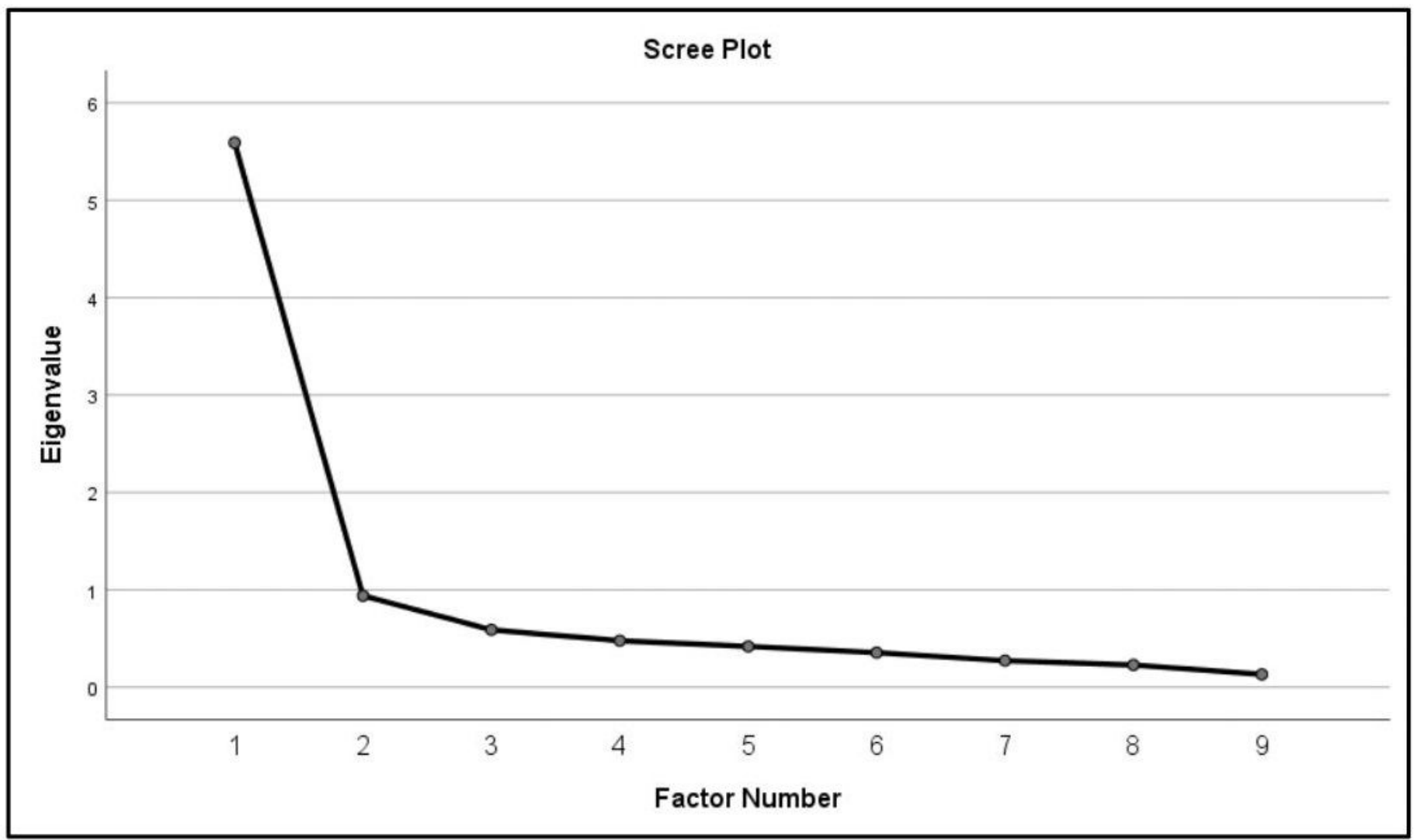

Figure 3

Scree plot resulted from principal factor analysis (PFA) in attitude domain 


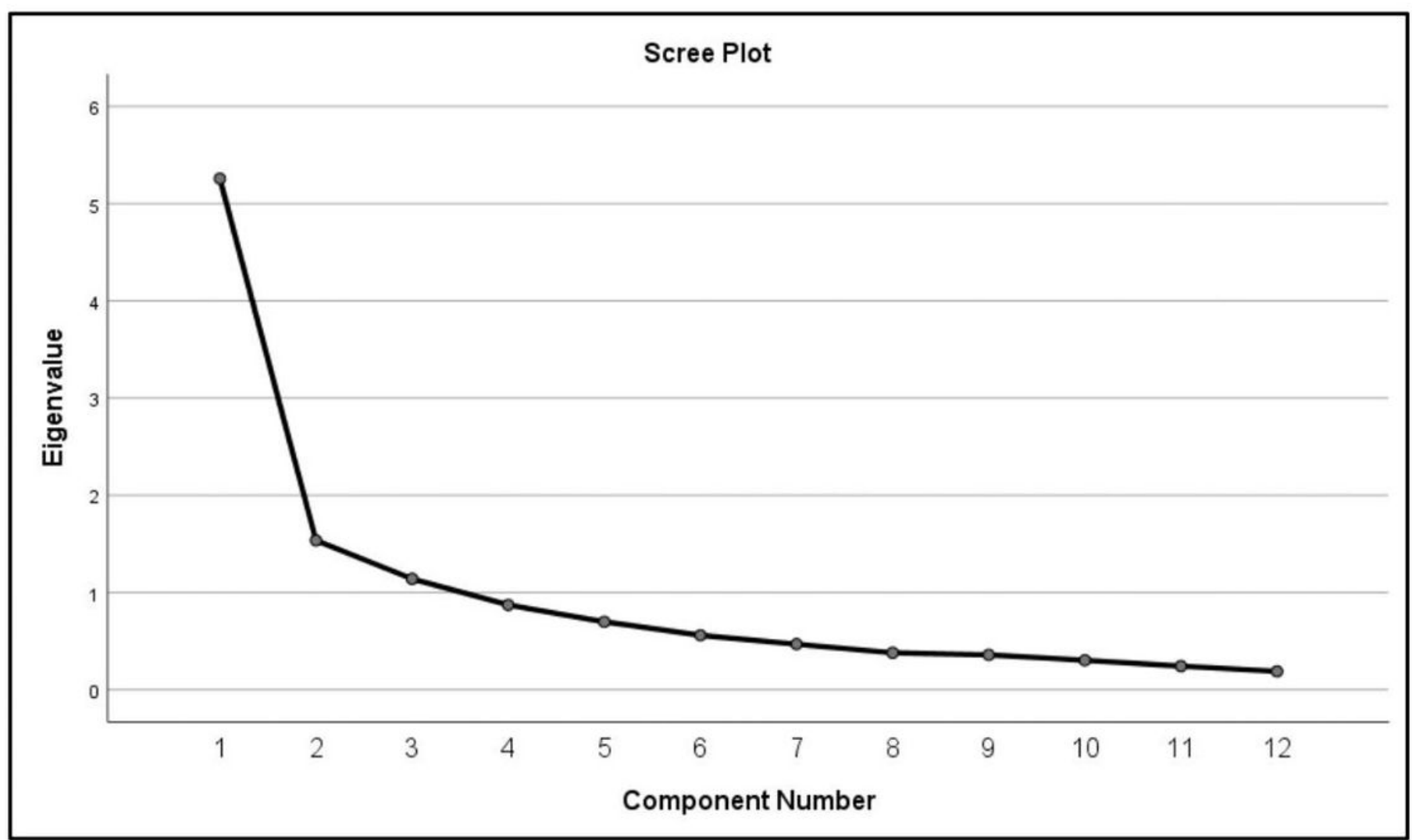

Figure 4

Scree plot resulted from principal factor analysis (PFA) in knowledge domain

\section{Supplementary Files}

This is a list of supplementary files associated with this preprint. Click to download.

- KAPPI.docx 